\title{
LONG RANGE SCATTERING WITH STARK EFFECT AND ALMOST PERIODIC POTENTIALS
}

\author{
DENIS A.W. WHITE
}

Department of Mathematics,

University of Toledo,

Toledo, OH 43606

Received December 3, 1990 and revised August 20, 1991

\begin{abstract}
Spectral and scattering theory is discussed for the Stark effect Hamiltonians $H_{0}=$ $-(1 / 2) \Delta-x_{1}$ and $H=H_{0}+V$ where $V$ is a long range perturbation. Most significantly, in one dimension, and for $V$ consisting of a slowly decaying term and an almost periodic term, the two Hilbert space wave operators (of Isozaki and Kitada) are shown to exist and be complete by Enss's time dependent method.
\end{abstract}

Key Phrases. Long range scattering, Stark effect, almost periodic, two Hilbert space wave operators.

1991 AMS Subject Classification. Primary 35P25; Secondary 47A40, 81 U05.

\section{Introduction}

This note concerns quantum mechanical scattering in the presence of a constant electric field. The corresponding "Stark effect" Hamiltonians are,

$$
\begin{gathered}
H_{0}=-\frac{1}{2} \Delta-x_{1} \text { where } \Delta=\sum_{j=1}^{n} \partial^{2} / \partial x_{j}^{2} \\
H=H_{0}+V=H_{0}+V_{L 1}+V_{L 2}+V_{S}
\end{gathered}
$$

where $-x_{1}$ is the potential corresponding to the constant electric field in the positive $x_{1}$ direction $\left(x \in \mathbf{R}^{n}\right)$ and $H$ is a perturbation of $H_{0}$ by another potential $V=V_{L 1}+V_{L_{2}}+V_{S}$ consisting of two long range terms and a short range term. More precisely assume

Conditon LR1. $V_{L 1} \in C^{\infty}\left(\mathbf{R}^{n}\right)$ is real valued and for some $\epsilon>0$

$$
\left|D^{\alpha} V_{L_{1}}(x)\right|<C_{\alpha}\left\langle x_{1}\right\rangle^{-|\alpha| / 2-e} \text { for every multi-index } \alpha
$$

where $\left\langle x_{1}\right\rangle^{2}=\left(1+x_{1}^{2}\right)$.

Conditon LR2. $V_{L 2} \equiv 0$ if the space dimension is $n>1$ but if $n=1$ then $V_{L 2} \in C^{\infty}(\mathbf{R})$ is real valued and bounded along with all its derivatives and

$$
\int_{1}^{\infty} V_{L 2}\left(x+\frac{1}{2} \tau^{2}\right) d \tau
$$


exists as an improper Riemann integral for every $x$.

Condition SR. $V_{S}$ is a symmetric operator which is $H_{0}$-compact and

$$
\int_{1}^{\infty}\left\|F\left(x_{1}>r^{2}\right) V_{S}\left(H_{0}+i\right)^{-1}\right\| d r<\infty
$$

where $F(\cdot)$ is multiplication by the characteristic function of the indicated set.

It should be clarified that the term $V_{L 2}$ which allows an almost periodic potential is only present in the one dimensional case. Much of the recent work has focused on the one dimensional case; see Hislop-Nakamura [1], Jensen [2], Jensen-Yajima [3] and Ozawa [4] for example. The methods here are primarily multi-dimensional and the role of the assumption $n=1$ will be highlighted below. Almost periodic potentials and the existence and completeness of the ordinary wave operators (of equation (1.6) below) have been studied previously [2]. Long range scattering in this setting is relatively new but there has been some interest recently because there is a discrepancy between quantum and classical mechanics; the ordinary wave operators exist in the classical setting but not the quantum one when $n=1$ and $V_{L 2}=0$ as was noted recently by Jensen-Yajima [3] and Jensen-Ozawa [5]. In Theorem 2 below an additional condition on the potential is introduced which assures that the two Hilbert space wave operators equal the ordinary wave operators. In general one expects that the two Hilbert space wave operators are equal, up to a phase function, to the Dollard's modified wave operators; the justification of this expectation will be the subject of a future investigation. That result and Theorem 1 below will precisely delineate for which potentials of those considered here, the usual wave operators exist and are complete. For the potentials $V_{L 1}$ the condition is $\epsilon>1 / 2$ (see Jensen-Yajima [3] and Ozawa [4] when $n=1$ ) but for $V_{L 2}$ the condition is not obvious; see Theorem 2.

The main result is the existence and completeness of the two Hilbert space wave operators, $W^{ \pm} \equiv W^{ \pm}\left(H, H_{0} ; J^{ \pm}\right)$defined by

$$
W^{ \pm}=s-\lim _{t \rightarrow \mp \infty} e^{i t H} J^{ \pm} e^{-i t H_{0}}
$$

where $J^{ \pm}$are two bounded operators on $L^{2}\left(\mathbf{R}^{n}\right)$, to be specified and "s-lim" is the limit in the strong operator topology. Note the reversal of sign in equation (1.3) which is for historical reasons (see Reed and Simon's third volume [6, p. 17]). The two operators $J^{ \pm}$could equally well be replaced by a single operator as was previously noted by the author [7][equation (2.10)] and as is customary in most discussions of two Hilbert space scattering. (The operators $J^{ \pm}$are not unique.) Isozaki and Kitada [8] were the first to introduce $J^{ \pm}$("time independent modifiers") in this context; they are defined by

$$
J^{ \pm} \phi(x)=\int e^{i x \cdot \xi+i \theta^{ \pm}(x, \xi)} \hat{\phi}(\xi) d_{1} \xi
$$

where $\hat{\phi}$ is the Fourier transform of $\phi, d_{1} x=(2 \pi)^{-n / 2} d x$ and $\theta^{ \pm}$are functions to be chosen. The functions $\theta^{ \pm}$will be specified in $\S 3$ below but roughly they are chosen so that the commutator $H J^{ \pm}-J^{ \pm} H_{0}$ is small. (Recall Cook's method.) The symbol of $H J^{ \pm}-J^{ \pm} H_{0}$ is, at least in the case that $V_{S}=0$ :

$$
\begin{aligned}
p^{ \pm}(x, \xi)= & \xi \cdot \nabla_{x} \theta^{ \pm}(x, \xi)+\frac{\partial}{\partial \xi_{1}} \theta^{ \pm}(x, \xi)-\frac{i}{2} \Delta_{x} \theta^{ \pm}(x, \xi) \\
& +\frac{1}{2} \nabla_{x} \theta^{ \pm}(x, \xi) \cdot \nabla_{x} \theta^{ \pm}(x, \xi)+V_{L 1}(x)+V_{L 2}(x)
\end{aligned}
$$

and this should be small; that is roughly "short range" when $\mp \xi_{1}>0$. The main results may now be stated. 
Theorem 1 Assume the Conditions LR1, LR2 and SR. Then for $\mathrm{J}^{ \pm}$as defined above (for appropriate $\theta^{ \pm}$), the wave operators $W^{ \pm}$of (1.3) exist, are isometries and are complete. Moreover $H$ has no singularly continuous spectrum, and its eigenvalues are discrete and of finite multiplicity.

Theorem 2 Under the hypotheses of Theorem 1 and in the special case $n=1, V_{L 1}=0$ and

$$
\lim _{x, \xi \rightarrow \infty} \int_{\xi}^{\infty} V_{L 2}\left(x-\frac{1}{2} \xi^{2}+\frac{1}{2} \tau^{2}\right) d \tau=0
$$

it is possible to choose $J^{ \pm}$to be the identity operator in Theorem 1, that is

$$
W^{ \pm}=s-\lim _{t \rightarrow \mp \infty} e^{i t H} e^{-i t H_{0}} .
$$

Examples. The conditions of Theorem 1 are verified if: for $\epsilon>0$ and $\alpha, \beta \leq 1 / 2$ and $1 \geq \gamma>1 / 2$ and $b_{1}, b_{2}, b$ are real,

$$
\begin{aligned}
& V_{L 1}(x)=\langle x\rangle^{-\epsilon} \cos \left(b_{1}\left|x_{1}\right|^{\alpha}\right) \cos \left(b_{2}|x|^{\beta}\right) \\
& V_{L 2}(x)=\sin \left(b x^{\gamma}\right)+U^{\prime}(x)
\end{aligned}
$$

where $U$ is a real function which is bounded along with all its derivatives (but $V_{L_{2}} \equiv 0$ if $n>1$.) The conditions of Theorems 1 and 2 are linear and so the set of potentials covered by these results form a linear space. For examples of the short range potentials $V_{S}$ see Yajima [9]. Roughly $V_{S}$ should be $O\left(\left\langle x_{1}\right\rangle^{-1 / 2-c}\right)$ for $x_{1}>0$ and some $\epsilon>0$ and $o\left(\left\langle x_{1}\right\rangle\right)$ for $x_{1}<0$.

One simple example indicates that there is indeed a difference between one and more dimensions: for $V\left(x_{1}, x_{2}\right)=\sin x_{1}+\sin x_{2}$ and $n=2$ the wave operators (1.6) do not exist by a tensor product argument whereas they do if $V_{L_{2}}(x)=\sin x$ when $n=1$. If $V_{L_{2}}(x)=\sin \left(x^{2 / 3}\right)$ then Theorem 1 applies but not Theorem 2. Jensen, [2] using Mourre's [10] method obtains a result similar to Theorem 2 for a class of real valued almost periodic potentials,

$$
V_{L 2}(x)=\int_{-\infty}^{\infty} e^{i x \xi} d \mu(\xi) \text { provided } \int_{-\infty}^{\infty} \xi^{2}+\xi^{-2} d \mu(\xi)<\infty
$$

(and with $J^{ \pm} \equiv 1$ and $V_{L 1}=0$ ). Theorem 2 requires more differentiability but one less antiderivative; more precisely, for each $N \geq 0$

$$
\int_{-\infty}^{\infty}|\xi|^{-1}+\xi^{2 N} d \mu(\xi)<\infty
$$

Theorem 1, which includes both slowly decaying and almost periodic potentials, is new.

\section{Two Hilbert Space Scattering.}

The derivation of Theorem 1.1 is broken into two main steps. The first step (Theorem 2.1 below) is to derive necessary conditions on $J^{ \pm}$or, in view of (1.4), conditions on $\theta^{ \pm}$to conclude Theorem 1.1. The second step (in $\S 3$ ) is to construct $\theta^{ \pm}$satisfying those conditions, from the long range potential $V_{L}$. Portions of the proofs will be cited from White [7] which will be quoted frequently simply as [W] for brevity's sake. More general potentials $V_{L 2}$ are allowed in the one dimensional case because

$$
\left.F\left(\left|D_{1}\right|<r\right)\right)\left(H_{0}+i\right)^{-1} \text { is compact if } n=1 .
$$


since it is unitarily equivalent via $\exp \left(-i D_{1}^{3} / 6\right)$ to a Hilbert Schmidt operator (see Perry [11, Proposition 19.1]); here $D,=-i \partial / \partial x_{j}$.

Before stating the conditions on $\theta^{ \pm}$it is convenient to recall the Calderón-Vaillancourt theorem which will be required to show that $J^{ \pm}$and related operators are bounded. Introduce therefore the pseudo-differential operator $R$

$$
R \phi(x)=\text { Os- } \iint e^{i \xi \cdot(x-y)} \rho(x, y, \xi) \phi(y) d x d \xi
$$

for all $\phi \in \mathcal{S}\left(\mathbf{R}^{n}\right)$. Here "Os-" indicates that the integrals are oscillatory integrals (see Kumanago [12]). The theorem can be stated in terms of certain norms on the symbol, $\rho$ : for each integer $k$

$$
|\rho|_{k} \equiv \sup \left\{\left|D_{x}^{\alpha} D_{\xi}^{\beta} \rho(x, y, \xi)\right|,\left|D_{y}^{\gamma} D_{\xi}^{\beta} \rho(x, y, \xi)\right|\right\}
$$

where the sup is over all $(x, y, \xi) \in \mathbf{R}^{3 n}$ and all $\alpha, \beta$, $\gamma$ so that $0 \leq|\alpha|,|\gamma| \leq 2 m$ and $0 \leq|\beta| \leq$ $2([n / 2]+k+1)$ and where $m$ is the least integer $m \geq 5 n / 4$. The Calderón-Vaillancourt theorem [13] says that there is a constant $C$ not depending on $\rho$ so that

$$
\|R\| \leq C|\rho|_{0}
$$

where $\|\cdot\|$ denotes the operator norm on $L^{2}\left(\mathbf{R}^{n}\right)$.

Anticipating the use of Enss's time dependent method ([14]) we introduce smooth versions of the incoming and outgoing operators, $F\left(D_{1}<0\right)$ and $F\left(D_{1}>0\right)$ respectively. Choose $\eta$ in $C^{\infty}(\mathbf{R})$ so that

$$
\eta\left(\xi_{1}\right)=\left\{\begin{array}{ll}
1 & \text { if } \xi_{1}>1, \\
0 & \text { if } \xi_{1}<-1
\end{array} \text { and } \eta\left(\xi_{1}\right)+\eta\left(-\xi_{1}\right)=1 .\right.
$$

Then the smooth versions of the incoming and outgoing operators are $\eta\left(-D_{1}\right)$ and $\eta\left(D_{1}\right)$ respectively. Define further $\eta_{1}\left(\xi_{1}\right)=\eta\left(\xi_{1}-2\right)$ so that

$$
\eta_{1}\left(\xi_{1}\right)= \begin{cases}1 & \text { if } \xi_{1}>3 \\ 0 & \text { if } \xi_{1}<1\end{cases}
$$

The hypotheses on $\theta^{ \pm}$can now be stated, beginning with $\theta^{-}$for simplicity. The first assumptions are technical: $\theta^{-} \in C^{\infty}\left(\mathbf{R}^{n} \times \mathbf{R}^{n}\right)$, is complex valued and for all multi-indicies $\alpha$ and $\beta$ there are constants $C>0$, and $N>0$ so that

$$
\begin{aligned}
\left|D_{x}^{\alpha} D_{\xi}^{\beta} \theta^{-}(x, \xi)\right| & <C \quad \text { provided }|\alpha| \geq 1 ; \\
\left|D_{\xi}^{\beta} \frac{\partial}{\partial \xi_{1}} \theta^{-}(x, \xi)\right| & <C\left(\langle\xi\rangle+\langle\xi\rangle^{\beta}\right) ; \\
\left|D_{\xi}^{\beta} \theta^{-}(x, \xi)\right| & <C(1+|x|)(1+|\xi|)^{N} \\
\left|\partial_{x} \partial_{\xi} \theta^{-}\right|_{m} & <1 / 2 .
\end{aligned}
$$

where $\langle\xi\rangle^{2} \equiv\left(1+|\xi|^{2}\right)$ and $m$ is the least integer $m \geq 5 n / 4$. Now define $p^{-}$by (1.5). The short range assumption is

$$
\int_{1}^{\infty}\left|\eta_{1}\left(x_{1} / r^{2}\right) \eta_{1}\left(\xi_{1} / r\right) p^{-}\right|_{m} d r<\infty
$$

Further there are two compactness assumptions and here the dependence on dimension becomes apparent. Assume, for $n=1$

$$
\begin{aligned}
\lim _{r \rightarrow \infty}\left[\left|\eta_{1}(x / r) \eta_{1}(\xi / r) p^{-}\right|_{m}\right. & +\left|\eta_{1}(x / r) \eta_{1}(\xi / r) \partial \theta^{-} / \partial x\right|_{m+1} \\
& \left.+\left|\eta_{1}(x / r) \eta_{1}\left(\xi_{1} / r\right) \Im \theta^{-}\right|_{m}\right]=0
\end{aligned}
$$


where $\Im \theta^{-}$denotes the imaginary part of $\theta^{-}$. When $n=1$ we write $x$ for $x_{1}$ etc. If $n>1$ then a stronger assumption is needed:

$$
\begin{gathered}
\lim _{r \rightarrow \infty}\left[\left|\eta\left(\xi_{1}-a\right) \eta\left(x_{1}-b\right) \eta_{1}\left(\frac{|x|}{r}\right) p^{-}\right|_{m}+\left|\eta\left(\xi_{1}-a\right) \eta\left(x_{1}-b\right) \eta_{1}\left(\frac{|x|}{r}\right) \nabla_{x} \theta^{-}\right|_{m+1}\right. \\
\left.+\left|\eta\left(x_{1}-b\right) \eta\left(\xi_{1}-a\right) \eta_{1}\left(\frac{|x|}{r}\right) \Im \theta^{-}\right|_{m}\right]=0
\end{gathered}
$$

for all real $a, b$. Similar hypotheses hold for $\theta^{+}$but it is more convenient to define

$$
\theta^{+}(x, \xi)=-\overline{\theta^{-}(x,-\xi)} \text { so that } \overline{J^{+} \phi(x)}=J^{-}(\bar{\phi})(x)
$$

("time reversal") where the bar denotes complex conjugation. Under these assumptions we have the following theorem.

Theorem 1 Define $H_{0}$ by (1.1) and let $H=H_{0}+V_{L}+V_{S}$ where $V_{L}=V_{L}(x)$ is a real valued function which as an operator acts multiplicatively, is $H_{0}$-bounded, and has $H_{0}$-bound strictly less than 1 and where $V_{S}$ satisfies Condition $S R$. If $J^{ \pm}$and $p^{ \pm}$are as defined in equations (1.4) and (1.5) and if $\theta^{ \pm}$and $p^{ \pm}$satisfy the above hypotheses then the conclusions of Theorem 1.1 are valid.

Outline of the Proof. The proof is by Enss's time dependent method which here is adapted to a two Hilbert space setting appropriate for studying long range scattering. The method can be described as follows. Let $H$ be any self adjoint operator on $L^{2}\left(R^{n}\right)$ with spectral measure by $E$ (but $H_{0}$ is defined by (1.1)) and suppose $J^{ \pm}$be bounded operators. Assume further

Hypothesis H1. There is $a_{0}<-1$ so that, for all $a, a>a_{0}$

$$
\left((H+i)^{-1} J^{ \pm}-J^{ \pm}\left(H_{0}+i\right)^{-1}\right) \eta\left(\mp D_{1}-a\right) \text { are compact. }
$$

Hypothesis H2. For every compact real interval $I$, there is an integer $N$ so that

$$
\int_{1}^{\infty}\left\|E(I)\left(H J^{ \pm}-J^{ \pm} H_{0}\right) \eta_{1}\left(\mp D_{1} / r\right) \eta_{1}\left(x_{1} / r^{2}\right)\left(H_{0}+i\right)^{-N}\right\| d r<\infty .
$$

Hypothesis H3. The following operator is compact

$$
(H+i)^{-1}\left[J^{+} \eta\left(-D_{1}\right)\left(J^{+}\right)^{*}+J^{-} \eta\left(D_{1}\right)\left(J^{-}\right)^{*}-1\right](H+i)^{-1} .
$$

Hypothesis H4. $e^{i t H_{0}}\left(\left(J^{ \pm}\right)^{*} J^{ \pm}-1\right) e^{-i t H_{0}} \rightarrow 0$ weakly as $t \rightarrow \mp \infty$.

Enss method arguments apply to derive the present theorem from these hypotheses; see [W, Theorems 2.1 and 2.2]. The first two hypotheses correspond to the standard Enss assumptions for the short range case; $\mathrm{H} 3$ assures that the operators $J^{ \pm}$are "almost" unitary and $\mathrm{H} 4$ assures that the wave operators $W^{ \pm}$are isometries, if they exist.

Check therefore Hypotheses H1 through H4. Suppose $n=1$ since the case of $n>1$ was considered in [W]. Only the outgoing " $"$ " case is considered but see (2.11).

Let $P=\left(H_{0}+V_{L}\right) J^{-}-J^{-} H_{0}$ so that

$$
P \phi(x)=\int e^{i x \xi+i \theta^{-}(x, \xi)} p^{-}(x, \xi) \phi(\xi) d_{1} \xi
$$

for $p^{-}$defined by (1.5). Since $V_{S}$ is $H_{0}$-compact by Condition $S R$, it suffices to show that $(H+$ $i)^{-1} P\left(H_{0}+i\right)^{-1} \eta\left(D_{1}-a\right)$ is compact for all $a$ to verify $H 1$. Since $1-\eta_{1}(D / r)$ is $H_{0}$-compact for 
any $r>0$, by (2.1), this may be simplified to showing that $(H+i)^{-1} P \eta_{1}(|D| / r)\left(H_{0}+i\right)^{-1} \eta(D-a)$ can be made arbitrarily close to a compact operator by choosing $r$ large enough. The proof of this latter statement is the same as the proof of $\mathrm{Hl}$ given in [W] but $P$ there is replaced by $P \eta_{1}(|D| / r)$. (It should be remarked that [W, Proposition 3.2 (a) and (b)], which is used in the proof cited above, assumes that $\theta^{ \pm}$is real valued but the proofs apply without change to the complex valued case provided the factor $\exp \left(-\Im \theta^{ \pm}\right)$appearing in the Fourier integral operators is treated as part of the symbol (and not the phase) as in the proof of $\mathrm{H} 3$ below.

The proof of $\mathrm{H} 2$ is exactly as in [W]; it uses the critical short range assumption (2.8) and the Calderón-Vaillancourt theorem.

To verify $\mathrm{H} 3$, it suffices to show that

$$
(H+i)^{-1}\left[J^{ \pm} \eta(\mp D)\left(J^{ \pm}\right)^{*}-\eta(\mp D)\right](H+i)^{-1} \text { are compact }
$$

because the sum of these two operators is the operator in H3. The argument is given for $\mathrm{J}^{-}$only; the $J^{+}$case is similar (or use (2.11)). First, by Hypothesis $\mathrm{Hl}, H_{0}$ is roughly " $J^{-}$-subordinate" to $H$ or more precisely

$$
\lim _{s \rightarrow \infty}\left\|(H+i)^{-1} J^{-} \eta(D) E_{0}(|\lambda|>s)\right\|=0
$$

by [W, Lemma 2.5]. This and (2.1) reduces (2.12) to showing

$$
(H+i)^{-1}\left[J^{-} \eta_{1}(D / r)\left(J^{-}\right)^{*}-\eta(D / r)\right](H+i)^{-1} \text { are compact }
$$

for all $r>0$. (Note $H$ and $H_{0}$ have to same domain and so may be interchanged in (2.1).) The argument of $[\mathrm{W}]$ applies (see also [W, Proposition 3.2(c)]). The change of variables in that argument involves only the real part $\Re \theta^{-}$of $\theta$ :

$$
\xi^{\prime}=\xi^{\prime}(\xi, x, y)=\xi+\int_{0}^{1} \nabla_{x} \Re \theta(y+s(x-y), \xi) d s .
$$

and uses assumption (2.9) but the proof is essentially the same.

The following theorem will be used for the proof of $\mathrm{H} 4$ and Theorem 1.2 both.

Theorem 2 Let $\tilde{\theta}^{-}$be a complex valued function on $\mathbf{R}^{2 n}$ satisfying (2.7) and with $\Im \tilde{\theta}^{-}$ bounded. Let $\tilde{J}^{-}$be defined by (1.4) with phase function $\theta^{-}$there replaced by $\tilde{\theta}^{-}$. If $m$ is the least integer $m \geq 5 n / 4$ and if

$$
\lim _{r \rightarrow \infty}\left|\eta_{1}\left(x_{1} / r^{2}\right) \eta_{1}\left(D_{1} / r\right)\left(\theta^{-}(x, \xi)-\tilde{\theta}^{-}(x, \xi)\right)\right|_{m}=0
$$

then

$$
s-\lim _{t \rightarrow \infty}\left(J^{-}-\tilde{J}^{-}\right) e^{-i t H_{0}}=0
$$

Proof of Theorem 2.2. By a density argument it suffices to prove the strong convergence on states of the form, $\eta\left(D_{1}-a\right) \eta\left(x_{1}-b\right) \phi$. Recall that outgoing states evolving freely constantly accelerate in the direction of the electric field except for an error term:

$$
\left\|\left(1-\eta_{1}\left(16 x_{1} / t^{2}\right)\right) e^{-i t H_{0}} \eta\left(D_{1}-a\right) \eta\left(x_{1}-b\right)\right\|=O\langle t\rangle^{-N}
$$

(see Perry [11, Lemma 19.7]). Also momentum is translated in the same direction:

$$
e^{-i t H_{0}} \eta\left(D_{1}-a\right)=\eta\left(D_{1}-a-t\right) e^{-i t H_{0}}
$$


by [11, Proposition 19.1] of Perry's book. Thus it is enough to show that

$$
\lim _{t \rightarrow \infty}\left\|\left(J^{-}-\tilde{J}^{-}\right) \eta_{1}\left(16 x_{1} / t^{2}\right) \eta\left(D_{1}-a-t\right)\right\|=0 .
$$

and this is follows from the assumptions on $\theta-\tilde{\theta}^{-}$(see [W, Proposition 3.2 (a) and (b)]).

To complete the proof of Theorem 1, it remains to check Hypothesis H4. By Theorem 2, and assumption (2.9) make it possible to assume $\theta^{-}$is real valued and in this case the proof in [W] applies. (There is an error in the proof just before equation (3.23) [W]: the definitions of $q_{1}$ and $q_{3}$ have been interchanged.)

Remark. To extend the above proof to $n$-dimensions, one would need a criterion, replacing (2.1) when $n>1$, for a pseudo-differential operator like $R$ in (2.2) to be compact with respect to $H_{0}$ which does not require the symbol to decay to 0 as $x_{1} \rightarrow \infty$.

\section{Theorem 1.1.}

The proof of Theorem 1.1 involves simply choosing $\theta^{-}$and checking the hypotheses of Theorem 2.1. The short range assumption (2.8) is the crucial to the choice.

Outline of the Proof of Theorem 1.1. Again it suffices to consider the $n=1$ and the outgoing "-" case. Moreover the construction of $\theta^{-}$(and thereby $\mathrm{J}^{-}$) was carried out in [W] in the case when $V_{L 2}=0$ and $n \geq 1$. Denote that function now as $\theta_{1}^{-}$. Next we construct $\theta_{2}^{-}$ as the appropriate choice of $\theta^{-}$for general $V_{L 2}$ and $V_{L 1}=0$ (and $n=1$ ); then the appropriate choice of $\theta^{-}$in the general case will turn out to be simply $\theta^{-}=\theta_{1}^{-}+\theta_{2}^{-}$. Define

$$
\theta_{2}^{-}(x, \xi)=\int_{\xi}^{\infty} \eta_{1}(\rho \tau) V_{L 2}\left(x-\frac{1}{2} \xi^{2}+\frac{1}{2} \tau^{2}\right) d \tau-\frac{i}{2} \xi^{-2} \eta_{1}(\rho \xi) V_{L 2}(x)
$$

where $\rho$ is a small parameter to be fixed after checking property (2.7). The Condition LR2 assures that the integral exists as an improper Riemann integral and defines $\theta_{2}^{-}$to be a continuous function. In fact $\theta_{2}^{-}$is infinitely differentiable and the derivatives may be computed by differentiating under the integral sign because if the derivatives are computed formally by this formula then the resultant integrals converge locally uniformly. This convergence can be checked by integrating by parts, $V_{L 2}^{\prime}$ being integrated. The choice of $\theta_{2}^{-}$is made with the short range assumption (2.8) in mind. Compute

$$
\begin{gathered}
p^{-}(x, \xi) \\
=\quad V_{L 2}(x)\left[\left(1-\eta_{1}(\rho \xi)\right)+\frac{i}{2}\left(3 \xi^{-3} \eta_{1}(\rho \xi)-(2 \rho) \xi^{-2} \eta_{1}^{\prime}(\rho \xi)\right)\right]-\frac{1}{4} \xi^{-2} V_{L_{2}}^{\prime \prime}(x) \\
\frac{1}{2}\left[i \xi^{-2} \eta_{1}(\rho \xi) V_{L_{2}}^{\prime}(x)-\xi^{-1} \eta_{1}(\rho \xi) V_{L 2}(x)\right. \\
\left.+\int_{\xi}^{\infty}\left(\tau^{-2} \eta_{1}(\rho \tau)-\rho \tau^{-1} \eta_{1}^{\prime}(\rho \tau)\right) V\left(x-\frac{1}{2} \xi^{2}+\frac{1}{2} \tau^{2}\right) d \tau\right]^{2}+ \\
-\frac{i}{2} \int_{\xi}^{\infty}\left[\rho^{2} \tau^{-2} \eta_{1}^{\prime \prime}(\rho \tau)-3 \rho \tau^{-3} \eta_{1}^{\prime}(\rho \xi)+3 \tau^{-4} \eta_{1}(\rho \tau)\right] V\left(x-\frac{1}{2} \xi^{2}+\frac{1}{2} \tau^{2}\right) d \tau
\end{gathered}
$$

It is a routine matter of checking that $\theta_{2}^{-}$satisfies the hypotheses of Theorem 2.2 and it suffices to remark that in checking (2.7) one must choose $\rho$ sufficiently small; $\rho$ then remains fixed. To do the check of the same hypotheses for $\theta^{-}=\theta_{1}^{-}+\theta_{2}^{-}$there is only one nonlinear condition that requires checking and that is the short range condition (2.8). Since

$$
\frac{\partial}{\partial x} \theta_{1}=O\left(\langle x\rangle^{-c}\right) \text { and } \frac{\partial}{\partial x} \theta_{2}=O\left(\langle\xi\rangle^{-1}\right)
$$


(sce [W, equation 4.10]) this is easily checked.

Proof of Theorem 1.2. This Theorem follows directly from Theorem 2.3 if one chooses $\tilde{\theta^{-}}=0$ there.

Acknowledgement: This research was supported in part by a Faculty Research Fellowship awarded by the University of Toledo.

\section{References}

[1] Hislop, P.D. and Nakamura, S. Stark Hamiltonians with unbounded random potentials, preprint (1990).

[2] Jensen, A. Scattering theory for Hamiltonians with Stark effect, Ann. Inst. Henri Poincare Phys. Theor. 46 (1987), 383-395.

[3] Jensen, A. and Yajima, K. On the long range scattering for Stark Hamiltonians preprint (1990), IES, Aalborg.

[4] Ozawa, T. Non-existence of wave operators for Stark effect Hamiltonians, preprint (1989).

[5] Jensen, A. and Ozawa, T. Classical and quantum scattering for Stark Hamiltonians with slowly decaying potentials, preprint, IES, Aalborg.

[6] Reed, M. and Simon, B. Methods of Modern Mathematical Physics. III Scattering Theory, New York, Academic Press 1979.

[7] [W] White, D. The Stark effect and long range scattering in two Hilbert spaces, Indiana Univ. Math. J. 39, 517-546.

[8] Isozaki, I. and Kitada, H. Modified wave openators with time-independent modifiers, J. Fac. Sci. Univ. Tokyo Sect. IA 32 (1985), 77-104.

[9] Yajima, K. Spectral and scattering theory for Schrödinger operators with Stark-effect, J. Fac. Sci. Univ. Tokyo, Sec IA, 26 (1979), 377-390.

[10] Mourre, E. Link between the geometric and spectral transformation approaches in scattering theory, Commun. Math. Phys. 68 (1979), 91-94.

[11] Perry, P.A. Scattering theory by the Enss Method, Mathematical Reports, No.1, Harwood, New York, 1983.

[12] Kumano-go, H. Pseudo-Differential Operators, MIT Press, Cambridge, Mass., 1981.

[13] Calderón, A.P. and Vaillancourt, R. A class of bounded pseudo-differential operators, Proc. Nat. Acad. Sci. U.S.A. 69 (1972), 1185-1187.

[14] Enss, V. Asymptotic completeness for quantum mechanical potential scattering, I. Short range potentials, Commun. Math. Phys. 61 (1978), 285-291.

[15] Simon, B. Phase space analysis of simple scattering systems: extensions of some work of Enss, Duke Math. J. 46 (1979), 119-168. 


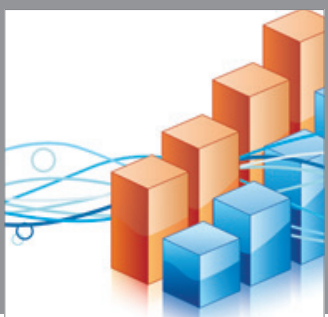

Advances in

Operations Research

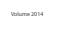

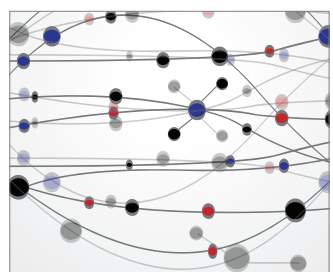

\section{The Scientific} World Journal
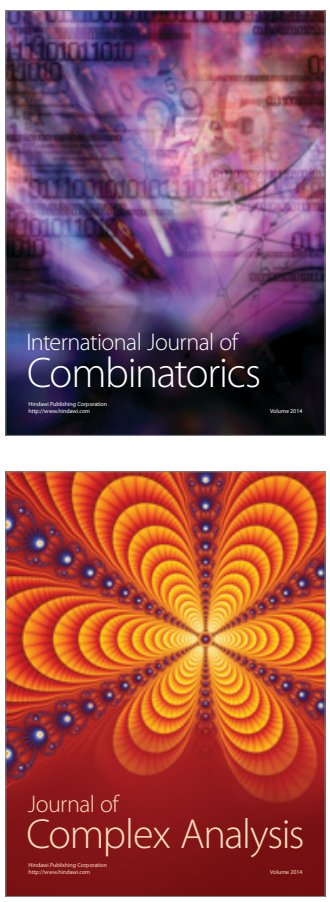

International Journal of

Mathematics and

Mathematical

Sciences
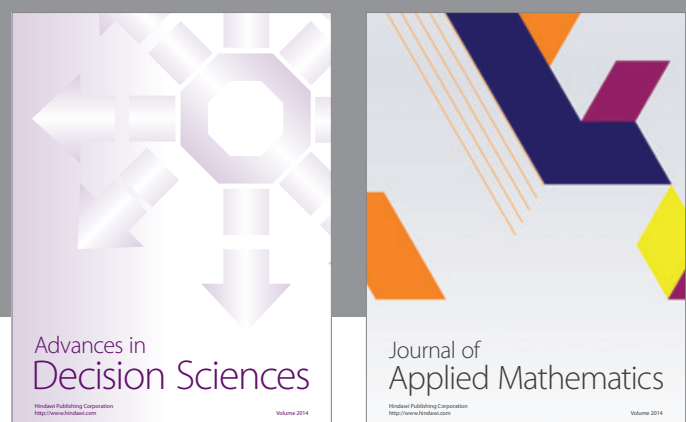

Journal of

Applied Mathematics
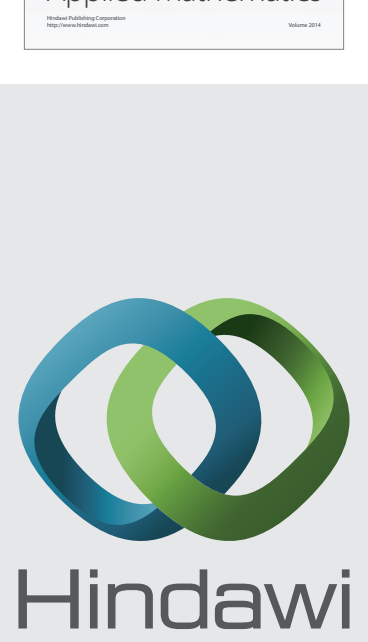

Submit your manuscripts at http://www.hindawi.com
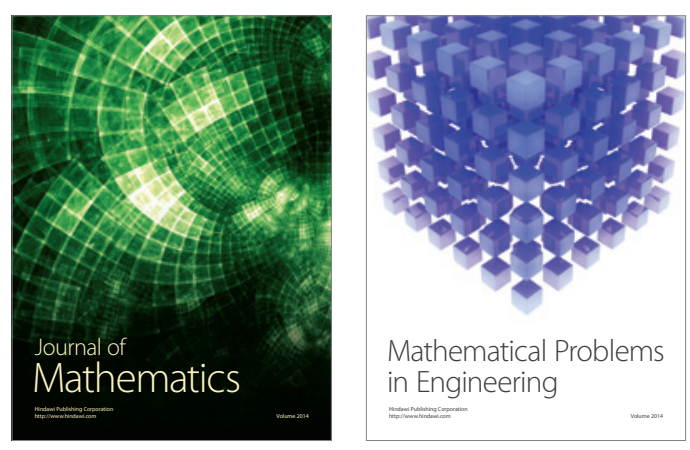

Mathematical Problems in Engineering
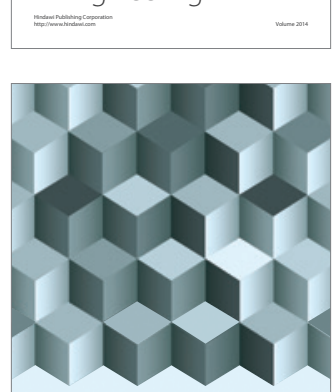

Journal of

Function Spaces
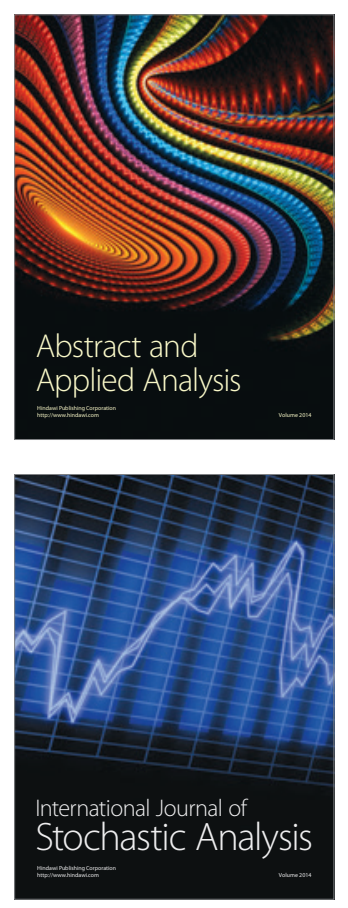

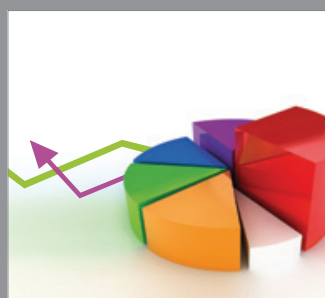

ournal of

Probability and Statistics

Promensencen
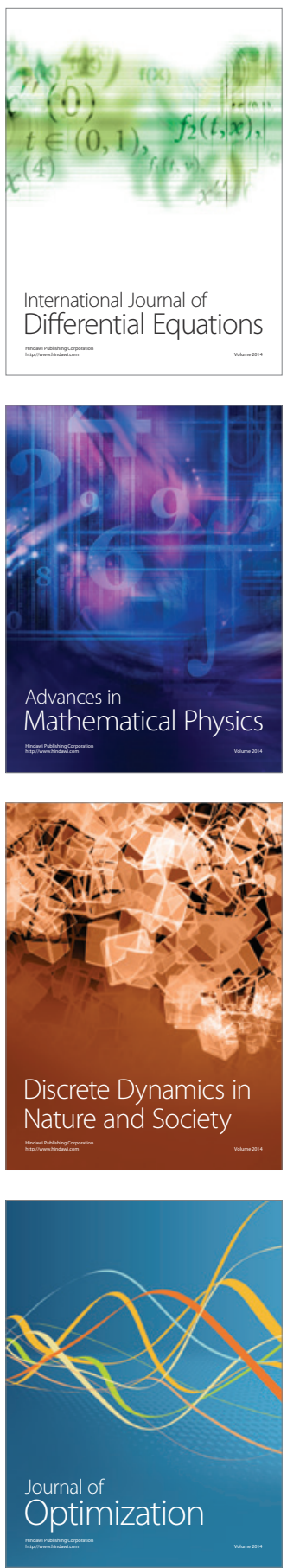